The Open Orthopaedics Journal
CrossMark
Content list available at: www.benthamopen.com/TOORTHJ/
DOI: $10.2174 / 1874325001610010111$

\title{
Combined Cubital and Carpal Tunnel Release Results in Symptom Resolution Outside of the Median or Ulnar Nerve Distributions
}

\author{
Peter C. Chimenti ${ }^{*}$, Allison W. McIntyre, Sean M. Childs, Warren C. Hammert and John C. Elfar \\ University of Rochester Medical Center, Rochester, New York, United States
}

Received: January 04, 2015

Revised: September 20, 2015

Accepted: November 18, 2015

\begin{abstract}
:
Background:

Resolution of symptoms including pain, numbness, and tingling outside of the median nerve distribution has been shown to occur following carpal tunnel release. We hypothesized that a similar effect would be found after combined release of the ulnar nerve at the elbow with simultaneous release of the median nerve at the carpal tunnel.
\end{abstract}

\section{Methods:}

20 patients with combined cubital and carpal tunnel syndrome were prospectively enrolled. The upper extremity was divided into six zones and the location of pain, numbness, tingling, or strange sensations was recorded pre-operatively. Two-point discrimination, Semmes-Weinstein monofilament testing, and validated questionnaires were collected pre-operatively and at six-week follow-up.

\section{Results:}

Probability of resolution was greater in the median nerve distribution than the ulnar nerve for numbness $(71 \% v s .43 \%)$, tingling ( $86 \%$ vs. $75 \%$ ). Seventy percent of the cohort reported at least one extra-anatomic symptom pre-operatively, and greater than $80 \%$ of these resolved at early follow-up. There was a decrease in pain as measured by validated questionnaires.

\section{Conclusion:}

This study documents resolution of symptoms in both extra-ulnar and extra-median distributions after combined cubital and carpal tunnel release. Pre-operative patient counseling may therefore include the likelihood of symptomatic improvement in a non-expected nerve distribution after this procedure, assuming no other concomitant pathology which may cause persistent symptoms. Future studies could be directed at correlating pre-operative disease severity with probability of symptom resolution using a larger population.

Keywords: Carpal Tunnel Syndrome, Cubital Tunnel Syndrome, Extra-Median, Extra-Ulnar, Median Nerve, Ulnar Nerve, Ulnar Neuropathy.

\section{INTRODUCTION}

The prevalence of carpal tunnel syndrome (CTS) has been reported to be between 3 and 5\% and is the most common compression neuropathy of the upper extremity [1]. Cubital tunnel syndrome (CuTS) is also quite common and represents the second most common cause of peripheral nerve compression [2]. The incidence of patients presenting with ipsilateral combined carpal and cubital tunnel syndrome is not known; however, it is the author's experience that the two diagnoses may frequently occur together. The diagnosis of either CuTS or CTS is made by a combination of clinical assessment with adjunctive electrodiagnostic studies (EDX) [3, 4]. Median nerve compression

\footnotetext{
* Address correspondence to this author at the Department of Orthopaedic Surgery 601 Elmwood Avenue, Box 665 Rochester, NY 14642, United States; Tel: 585.275.5117; Fax: 585.273-3297; E-mail: open_elfar@urmc.rochester.edu
} 
at the wrist typically produces numbness or parasthesias in the thumb, index, and middle finger [5] but can also cause pain in the radial three fingers or hand [6]. Compression of the ulnar nerve at the level of the elbow may result in parasthesias or numbness in the small finger and pain throughout the medial forearm from elbow to small finger [4, 7]. However, many patients with CuTS also report symptoms outside of the expected ulnar nerve distribution.

Improvement in subjective symptom reports both within and outside of the typical median nerve distribution after carpal tunnel release has been shown previously [8]. In 2009, Elfar and colleagues reported a prospective study in which the distribution of subjective complaints of patients undergoing carpal tunnel release was examined at early follow-up. They found a greater than ninety percent likelihood of symptom resolution outside of the median nerve distribution. Based on this data, we sought to investigate whether that a similar phenomenon would be found among patients undergoing combined ipsilateral concurrent cubital and carpal tunnel release. This study was designed to provide prognostic information to improve the quality of pre-operative guidance for patients with combined cubital and carpal tunnel syndrome.

\section{MATERIALS AND METHODS}

Institutional review board approval for this study was obtained prior to study initiation. Patients were considered eligible for the study if they met the following inclusion criteria: age greater than 18, clinical history and physical examination consistent with the diagnosis of combined cubital and carpal tunnel syndromes, EDX demonstrating slowing of ulnar nerve conduction across the elbow as well as median nerve conduction across the wrist. All EDX were performed by one electrophysiologist under standardized conditions. Exclusion criteria included any evidence of cervical radiculopathy, diabetes mellitus, diffuse polyneuropathy present on the EDX studies, or history of any prior surgical intervention for cubital or carpal tunnel syndrome at the medial elbow or wrist. Clinical diagnosis was made based on a history of numbness and/or parasthesias in the small finger exacerbated with prolonged elbow flexion in addition to numbness and/or parasthesias in the radial three fingers. Physical exam revealed positive Tinel's sign over the cubital tunnel and reproduction of cubital tunnel symptoms with elbow flexion. Patients also demonstrated a positive carpal tunnel compression test. A positive examination for all maneuvers was required for inclusion in this study. Although the literature does not support one single physical exam test as perfectly sensitive or specific for the diagnosis of carpal tunnel syndrome, we chose to use the carpal tunnel compression test as a means of standardizing our inclusion criteria. EDX studies consisting of nerve conduction and electromyographic components were routinely obtained to confirm the diagnosis and exclude co-existing pathology at other sites of potential compression including cervical nerve root or brachial plexus. Indication for surgical intervention included failure of non-operative treatment with persistent symptoms.

Patients were enrolled in clinic at their pre-operative visit. All procedures followed were in accordance with the ethical standards of the responsible committee on human experimentation (institutional and national) and with the Helsinki Declaration of 1975, as revised in 2008 (5). Informed consent was obtained from all patients for being included in the study. After a written informed consent process, patients were asked if they had regularly experienced four different symptom types throughout their operative extremity during the prior two weeks. The four symptoms were pain, numbness, tingling, and strange sensations that were clarified as burning or electrical type of sensations. Patient responses were indicated as presence or absence of the symptom at each location. The upper extremity was divided into 6 zones as shown in Fig. (1).

A physical examination was performed consisting of static two-point discrimination, tested with the MackinnonDellon Discriminator (Lafayette Instruments, Lafayette, Ind.) at all fingertips. Light touch sensation was tested at all digits using Semmes-Weinstein monofilaments (Smith \&amp; Nephew Roylan, Inc., Germantown, Wis.) Patients also completed questionnaires including the Boston Carpal Tunnel Questionnaire (CTQ) [9], the Michigan Hand Questionnaire (MHQ) [10], and the Visual Analogue Scale (VAS) [11]. The MHQ and CTQ scales were chosen as they have been previously validated for studying outcomes of both CTS and CuTS and are more responsive than other measures of upper extremity function [12]. At their six-week follow-up patients were asked to complete the same assessment of their symptom location and physical exam as well as questionnaires.

Descriptive statistics were used to calculate a mean and standard deviation for the number of zones with symptoms per patient. Further analysis revealed a non-normally distributed data set and therefore the Wilcoxon Signed-Rank test was used to evaluate for significant differences between means. Probability of symptom resolution in each zone was calculated by dividing the number of post-operative reports by pre-operative reports for each zone and symptom type. Differences in objective outcome data from both the questionnaires and 
physical examination data was analyzed using paired $t$-tests.

\section{RESULTS}

20 patients were enrolled in the study, the average age was 56 years (range $37-71$ ) and $50 \%$ of the patients were female. 17 of the 20 procedures performed were in situ decompression of which 11 were open and 6 endoscopic. The remaining 3 patients underwent anterior transposition. Indication for anterior transposition was any evidence of an unstable nerve with elbow flexion intra-operatively. Average duration to follow-up testing was 7 weeks (range 4-12 weeks) post-operatively.
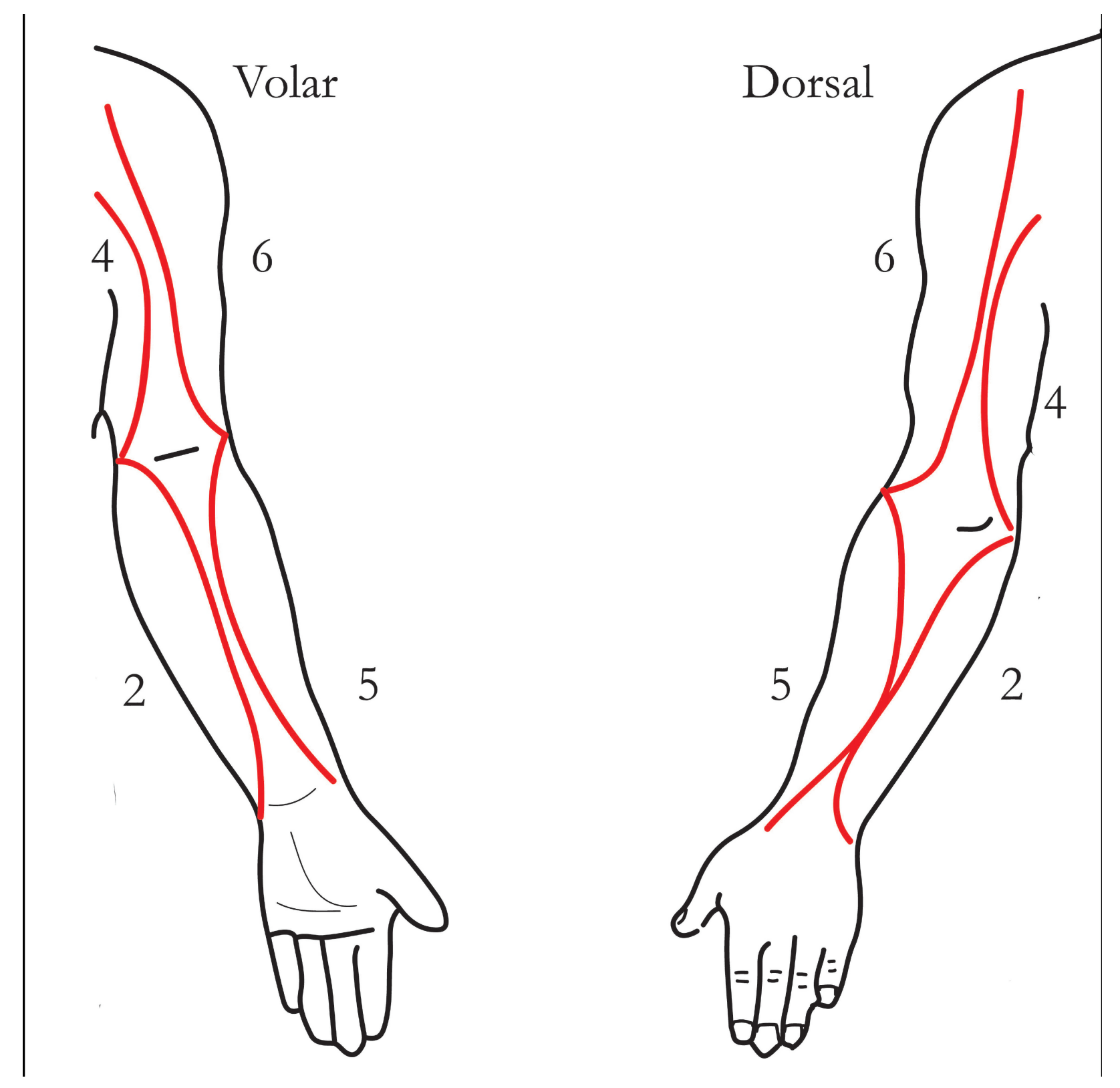


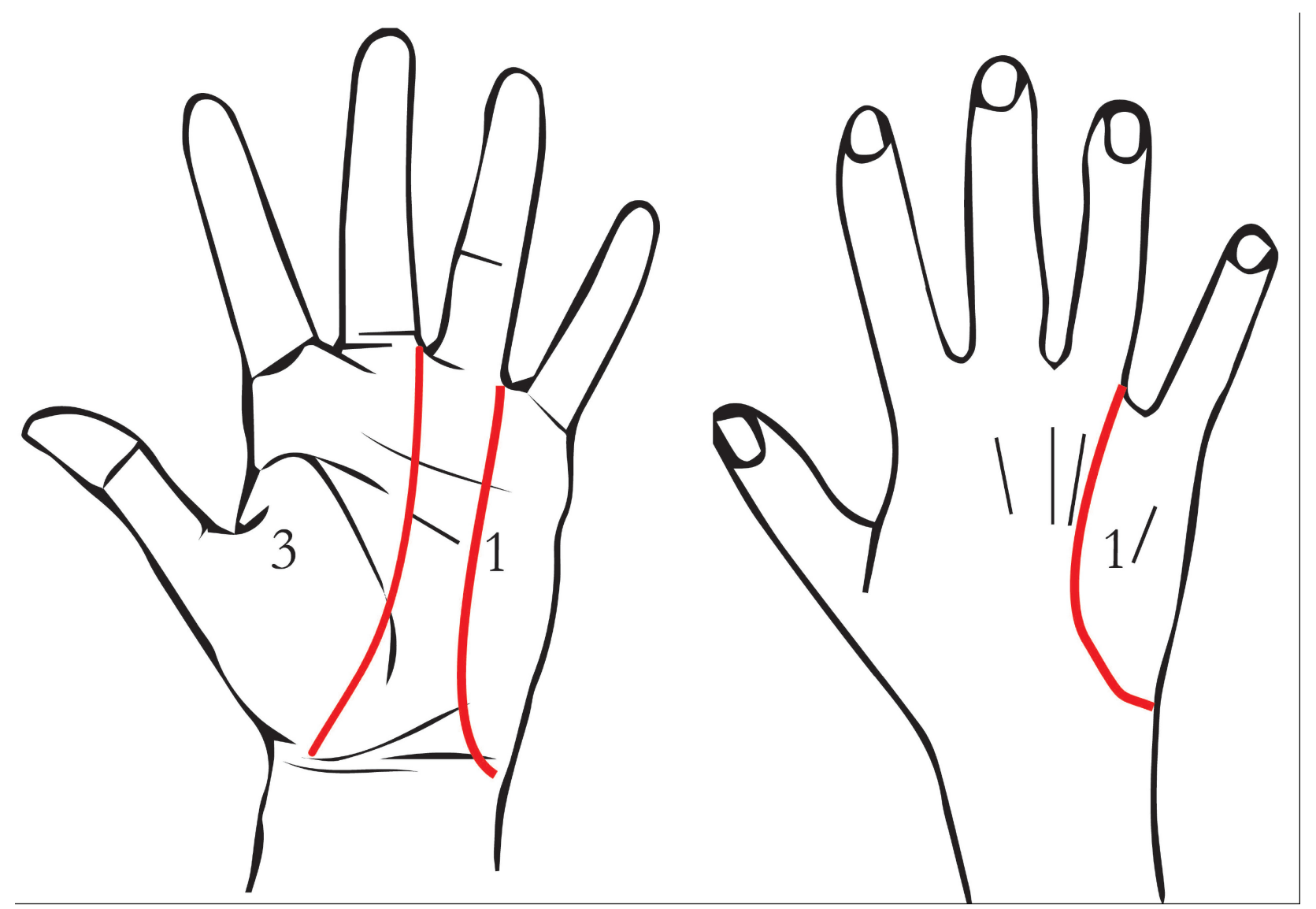

Fig. (1). Division of the upper extremity into six different zones. Note the exclusion of the ring finger due to dual innervation.

Combined concomitant ipsilateral cubital and carpal tunnel surgery demonstrated a high likelihood of symptom resolution both within and outside the expected median and ulnar nerve distribution. Table 1 shows the total pre- and post-operative symptom reports per zone as well as the probability of resolution for each symptom by zone. Roughly an equal number of reports were found in the ulnar (I) and median (III) zones pre-operatively, with 41 and 45 total reports, respectively. $68 \%$ of the cohort pre-operatively reported at least one symptom that was not referable to either the median or ulnar distributions (i.e. zones II, IV, V, VI). The median distribution showed a higher likelihood of resolution of numbness, 71\%, and parasthesias, 86\%, compared with the ulnar distribution (43 and 75\%) at early follow-up. However, there was also marked improvement in symptoms outside of either distribution with between a 75 and $100 \%$ likelihood of symptom resolution in zones II, IV, V, or VI. Interestingly, pain symptoms in particular were more likely to improve outside than within the expected median or ulnar distributions.

Table 1. Probability of symptom resolution by zone

\begin{tabular}{|l|l|l|l|l|l|l|}
\hline Symptom & Zone I & Zone II & Zone III & Zone IV & Zone V & Zone VI \\
\hline Total pre & 41 & 24 & 45 & 5 & 10 & 8 \\
\hline Total post & 17 & 5 & 11 & 0 & 1 & 0 \\
\hline Numbness & .43 & 1 & .71 & n/a & 1 & 1 \\
\hline Tingling & .75 & .83 & .86 & 1 & 1 & 1 \\
\hline Pain & .5 & .86 & .63 & 1 & .75 & 1 \\
\hline Strange & .6 & .4 & .83 & 1 & 1 & 1 \\
\hline
\end{tabular}

$\mathrm{n} / \mathrm{a}=$ not applicable (no reports occurred pre-operatively)

Total pre- and post-operative reports per zone shown in rows 1-2. Probability of resolution of each symptom by zone shown in rows 3-6.

To further analyze the zoned resolution of symptoms the extremity was divided into a surgical and non-surgical distribution. For the symptoms of numbness and tingling, the surgical distribution was defined as Zones I and III. For the symptoms of pain and strange sensations, the surgical distribution was defined as Zones I, II, III, and IV. We 
reasoned that inflammation within the ulnar nerve at the level of the cubital tunnel would be expected to cause radiating symptoms along the course of the nerve through the medial arm and forearm [4] and median nerve compression would be expected to cause symptoms referable to the radial three fingers (Zone III).

For each symptom type, the mean number of zones in which symptoms were reported was determined for both the surgical and non-surgical distributions. Shown in Table $\mathbf{2 A}$ are the results for the symptoms of numbness and tingling. Statistically significant resolution of symptoms was noted both within $(p=.003, p<.001)$ and outside of the surgical distribution $(p=.038, p=.016)$. As shown in Table 2B, pain symptoms were found to be significantly improved after surgery in the surgically released distribution $(p=.011)$ but failed to reach statistical significance outside of this area $(p=.059)$. Similarly, strange symptoms demonstrated statistically significant improvement in the surgical distribution $(p=.018)$ but did not reach significance elsewhere $(p=.059)$.

Table 2A. Mean number of zones with symptom reports pre- and post-op

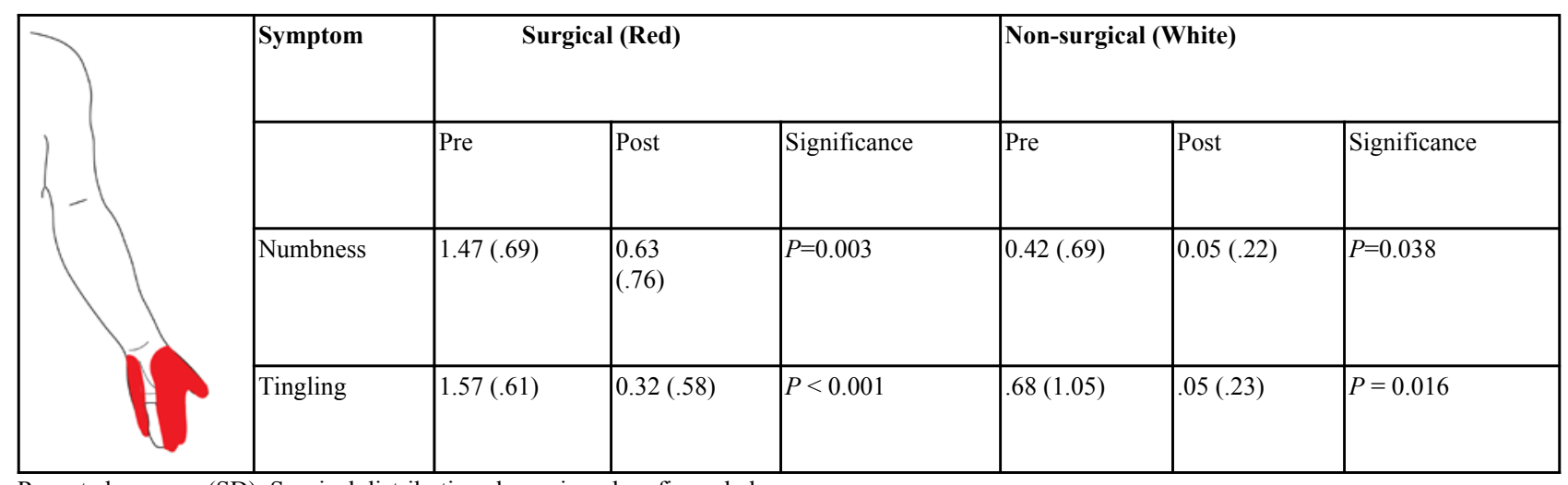

Reported as mean (SD). Surgical distribution shown in red on figure below.

Table 2B. Mean number of zones with symptom reports pre and postop

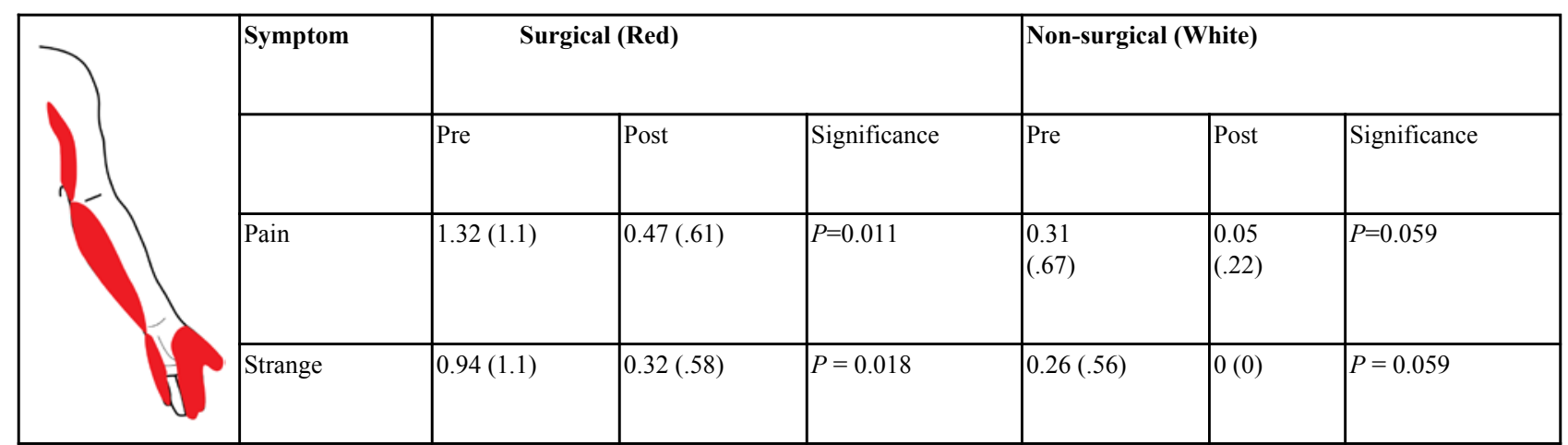

Reported as mean (SD). Surgical distribution shown in red on figure below.

There was significant improvement on the VAS from 70.6 pre-operatively to 24.4 post-operatively $(p<.001)$ (Table 3). Significant improvements were found in both the functional status scale and the symptom severity scales of the CTQ $(p<.001)$. The average MHQ score improved from 48 to 67 after surgery $(p<.001)$. Two-point discrimination showed significant improvement only at the middle finger post-operatively (6.43 to $4.57, p=.035$ ). Semmes-Weinstein monofilament data revealed a significant improvement in both index and small finger sensibility $(p=.017, p=.022)$ but failed to reach significance in the remaining digits.

\section{DISCUSSION}

In a prior study by the senior author, symptom resolution including numbness, tingling, pain, and strange symptoms was found to occur both within and outside the typical median nerve distribution [8]. In the present study we hypothesized that a similar result would be found to occur with patients undergoing combined carpal and cubital tunnel releases. Such information would have prognostic importance when counseling patients regarding expectations for symptom improvement after surgery for peripheral nerve entrapment. Our data suggest not only that a large percentage of patients with electrodiagnostic studies consistent with isolated CTS and CUTS also have symptoms outside of the typical ulnar or median nerve distributions; but moreover, that these symptoms have a high likelihood of resolving after 
surgery. Overall there was a greater than $75 \%$ probability of extra-territorial symptoms to improve (Table 1). Statistically significant decreases in the mean number of zones with symptoms was found for both numbness and tingling while pain and strange symptoms demonstrated a trend toward significance (Table $\mathbf{2 A} / \mathbf{2 B}$ ). There were fewer pre-operative reports of either of these two symptoms and as such there may have been insufficient numbers of reports to reach statistical significance.

Table 3. Patient-reported outcome measures.

\begin{tabular}{|l|l|l|l|}
\hline Questionnaire & Pre-operative & Post-operative & Significancee \\
\hline VASa & $70.6(21.3)$ & $24.4(20.9)$ & $\mathrm{p}<0.001$ \\
\hline FSSb & $2.69(1.07)$ & $1.75(.77)$ & $\mathrm{p}<0.001$ \\
\hline SSSc & $3.00(.89)$ & $1.75(.68)$ & $\mathrm{p}<0.001$ \\
\hline MHQd & $47.92(23.48)$ & $66.54(18.71)$ & $\mathrm{p}<0.001$ \\
\hline
\end{tabular}

Reported as mean (SD)

Reported as mean (SD)

$\mathrm{a}=$ Visual Analogue Scale

$\mathrm{b}=$ Functional Status Scale from the Boston Carpal Tunnel Questionnaire

$\mathrm{c}=$ Symptom Severity Scale from the Boston Carpal Tunnel Questionnaire

d= Michigan Hand Outcomes Questionnaire

$\mathrm{e}=$ Significance level determined using a paired t-test

Table 1 shows that pain symptoms were most likely to resolve in zones II, IV, V, and VI. This is in contrast to data our group has collected on isolated cubital tunnel patients who demonstrated complete relief of pain in zone III compared to the current cohort who demonstrated only $63 \%$ likelihood of improvement. In part this may be due to continued pain at the incision for carpal tunnel release that may be felt into the radial aspect of the hand. Longer followup of this cohort may be expected to demonstrate continued improvement of pain in this zone and thus better correlation with our earlier results; however, this cannot be confirmed with the results of this study.

Numbness and tingling within the ulnar distribution, zone I, were least likely to resolve at early follow-up, with only $43 \%$ and $75 \%$ probability of resolution, respectively. This is likely due to the early follow-up time point at which data was collected post-operatively. Prior studies have demonstrated continued improvement in patient-rated outcomes and physical examination measures for up to one year post-operatively [13] and the main objective of the present study was not to examine long-term outcome but to investigate symptom resolution in an extra-territorial distribution at early follow-up. Gilaldi and colleagues reported that significant improvements in the MHQ and CTQ are seen up to six months after surgery, and improvements in two-point discrimination may take 3 months to become significant and may continue for at least one year [13]. The present study found a significant improvement only in middle finger sensibility with two-point discrimination testing and index/small finger sensibility with Semmes-Weinstein testing. Interpretation of this data is somewhat limited by small sample size given that the study was not powered to detect differences in physical exam outcomes. Tactile sensory threshold has been shown to maximally improve between 10 and 30 days after carpal tunnel release [14]. Our finding of improvement in middle finger two-point discrimination is consistent with studies showing the greatest likelihood of improvement in the middle finger [5].

Interconnections between the median and ulnar nerves in the forearm, or Martin-Gruber connections, have been ruled out previously as a cause of extra-median spread of symptoms in CTS as they typically involve motor branches and rarely sensory pathways and have been shown with EDX studies to be absent in patients with extra-median symptoms [17]. Instead, spinal, subcortical, and cortical pathways have been implicated in the pathophysiology of symptomatic extra-anatomic peripheral neuropathy $[15,17]$. Tecchio and colleagues used magnetoencephalography to study cortical reorganization and plasticity in CTS patients with extra-median symptoms. They found that patients with parasthesias or pain demonstrated altered cortical representation of the affected hand in the contralateral hemisphere and they hypothesize that aberrant cortical reorganization may underlie persistent symptomatology in patients who fail to improve after carpal tunnel release [18]. NMDA-mediated central sensitization to afferent pain signals within the spinal cord as a result of increased release of spinal dynorphin has been observed in animal models after peripheral nerve injury that may also explain extraterritorial neuropathic pain $[16,19]$.

The present study has several limitations. Multiple surgical procedures were used in this study according to treating surgeon preference. We chose to include these because previous studies have indicated that similar outcomes can be expected after in situ, anterior transposition, and endoscopic techniques for cubital tunnel release [20]. Meta-analyses of 
different techniques have failed to identify any differences in outcomes after anterior transposition compared to simple decompression [21, 22]. Therefore we reasoned that inclusion of different approaches to cubital tunnel release would not alter the main outcome of extra-territorial symptom resolution.

The choice of surgical technique, whether endoscopic or open release, is unlikely to be a confounding factor for either carpal or cubital tunnel syndrome in terms of the primary outcome in this study. In a prospective trial, Watts and co-workers studied outcomes after endoscopic compared to open in situ release for cubital tunnel syndrome and found no significant differences at 12-month follow-up [23]. Although the literature is somewhat conflicting on the topic of endoscopic $v s$. open carpal tunnel release, a recent meta-analysis found similar subjective outcomes in terms of patient satisfaction and objective outcomes of hand and grip strength at 12-week follow-up [24]. Other studies, including a recent Cochrane database systematic review reported similar outcomes at early follow-up [25]. Therefore we reasoned that inclusion of both surgical techniques would not alter the primary outcome, namely patient-reported symptom resolution.

Another limitation of the study was the sample size, which was chosen to detect resolution of subjective symptom reports at early follow-up and was therefore not powered to look for differences in the degree of symptomatic resolution among patients with different pre-operative grades of disease severity. Indeed increased reports of extra-territorial symptoms were found among patients with milder CTS disease on neurographic studies [16] and therefore one might expect that patients with milder EDX studies would demonstrate a higher probability of resolution compared to more severe disease but this cannot be addressed with the current data.

Taken together the present data suggest that pre-operative counseling of patients with EDX-proven CTS and CuTS and symptoms outside of the typical median or ulnar nerve distribution should indicate a high likelihood ( $>80 \%)$ of resolution after combined cubital and carpal tunnel release, assuming that there are no other co-existing pathologies which may result in continued symptoms. Future studies could investigate any correlation between disease severity and likelihood of resolution, which would further improve the accuracy of pre-operative information that could be given to patients.

\section{LIST OF ABBREVIATIONS}

$\begin{array}{lll}\text { CTQ } & = & \text { Boston Carpal tunnel questionnaire } \\ \text { CTS } & = & \text { Carpal Tunnel Syndrome } \\ \text { CuTS } & = & \text { Cubital Tunnel Syndrome } \\ \text { EDX } & = & \text { Electrodiagnostic studies } \\ \text { MHQ } & = & \text { Michigan Hand Questionnaire } \\ \text { VAS } & = & \text { Visual analogue Scale }\end{array}$

\section{CONFLICT OF INTEREST}

The authors confirm that this article content has no conflict of interest.

\section{ACKNOWLEDGEMENTS}

The authors wish to thank Ruth L. Chimenti, DPT, PhD for assistance with appropriate statistical testing of research hypotheses.

\section{REFERENCES}

[1] Atroshi I, Gummesson C, Johnsson R, Ornstein E, Ranstam J, Rosén I. Prevalence of carpal tunnel syndrome in a general population. JAMA 1999; 282(2): 153-8. [http://dx.doi.org/10.1001/jama.282.2.153] [PMID: 10411196]

[2] Elhassan B, Steinmann SP. Entrapment neuropathy of the ulnar nerve. J Am Acad Orthop Surg 2007; 15(11): 672-81. [http://dx.doi.org/10.5435/00124635-200711000-00006] [PMID: 17989418]

[3] Bergquist ER, Hammert WC. Timing and appropriate use of electrodiagnostic studies. Hand Clin 2013; 29(3): 363-70. [http://dx.doi.org/10.1016/j.hcl.2013.04.005] [PMID: 23895716]

[4] Palmer BA, Hughes TB. Cubital tunnel syndrome J Hand Surg Am 2010; 35: 153-63. [http://dx.doi.org/10.1016/j.jhsa.2009.11.004] 
[5] Elfar JC, Yaseen Z, Stern PJ, Kiefhaber TR. Individual finger sensibility in carpal tunnel syndrome. J Hand Surg Am 2010 ; $35(11)$ : $1807-12$. [http://dx.doi.org/10.1016/j.jhsa.2010.08.013] [PMID: 21050964]

[6] Nora DB, Becker J, Ehlers JA, Gomes I. Clinical features of 1039 patients with neurophysiological diagnosis of carpal tunnel syndrome. Clin Neurol Neurosurg 2004; 107(1): 64-9.

[http://dx.doi.org/10.1016/j.clineuro.2004.08.003] [PMID: 15567555]

[7] Huang JH, Samadani U, Zager EL. Ulnar nerve entrapment neuropathy at the elbow: simple decompression. Neurosurgery 2004; 55(5): 1150-3. [http://dx.doi.org/10.1227/01.NEU.0000140841.28007.F2] [PMID: 15509321]

[8] Elfar JC, Calfee RP, Stern PJ. Topographical assessment of symptom resolution following open carpal tunnel release. J Hand Surg Am 2009; 34(7): 1188-92.

[http://dx.doi.org/10.1016/j.jhsa.2009.04.024] [PMID: 19643551]

[9] Levine DW, Simmons BP, Koris MJ, et al. A self-administered questionnaire for the assessment of severity of symptoms and functional status in carpal tunnel syndrome. J Bone Joint Surg Am 1993; 75(11): 1585-92.

[PMID: 8245050]

[10] Chung KC, Pillsbury MS, Walters MR, Hayward RA. Reliability and validity testing of the Michigan Hand Outcomes Questionnaire. J Hand Surg Am 1998; 23(4): 575-87. [http://dx.doi.org/10.1016/S0363-5023(98)80042-7] [PMID: 9708370]

[11] Williamson A, Hoggart B. Pain: a review of three commonly used pain rating scales. J Clin Nurs 2005; 14(7): 798-804. [http://dx.doi.org/10.1111/j.1365-2702.2005.01121.x] [PMID: 16000093]

[12] Song JW, Waljee JF, Burns PB, et al. An outcome study for ulnar neuropathy at the elbow: a multicenter study by the surgery for ulnar nerve (SUN) study group. Neurosurgery 2013; 72(6): 971-81. [http://dx.doi.org/10.1227/NEU.0b013e31828ca327] [PMID: 23426153]

[13] Giladi AM, Gaston RG, Haase SC, et al. Trend of recovery after simple decompression for treatment of ulnar neuropathy at the elbow. Plast Reconstr Surg 2013; 131(4): 563e-73e. [http://dx.doi.org/10.1097/PRS.0b013e318282764f] [PMID: 23542274]

[14] Wiacek R, Pielka S, Rutowski R, Gosk J, Skiba K, Reichert P. Evaluation of the dynamics of sensory improvement in the hand after surgical treatment of carpal tunnel syndrome. Neurol Neurochir Pol 2007; 41(6): 517-24. [PMID: 18224574]

[15] Tamburin S, Cacciatori C, Praitano ML, Marani S, Zanette G. Ulnar nerve impairment at the wrist does not contribute to extramedian sensory symptoms in carpal tunnel syndrome. Clin Neurophysiol 2009; 120(9): 1687-92. [http://dx.doi.org/10.1016/j.clinph.2009.07.001] [PMID: 19640785]

[16] Zanette G, Cacciatori C, Tamburin S. Central sensitization in carpal tunnel syndrome with extraterritorial spread of sensory symptoms. Pain 2010; 148(2): 227-36. [http://dx.doi.org/10.1016/j.pain.2009.10.025] [PMID: 20004060]

[17] Zanette G, Marani S, Tamburin S. Extra-median spread of sensory symptoms in carpal tunnel syndrome suggests the presence of pain-related mechanisms. Pain 2006; 122(3): 264-70. [http://dx.doi.org/10.1016/j.pain.2006.01.034] [PMID: 16530966]

[18] Tecchio F, Padua L, Aprile I, Rossini PM. Carpal tunnel syndrome modifies sensory hand cortical somatotopy: a MEG study. Hum Brain Mapp 2002; 17(1): 28-36.

[http://dx.doi.org/10.1002/hbm.10049] [PMID: 12203686]

[19] Malan TP, Ossipov MH, Gardell LR, et al. Extraterritorial neuropathic pain correlates with multisegmental elevation of spinal dynorphin in nerve-injured rats. Pain 2000; 86(1-2): 185-94. [http://dx.doi.org/10.1016/S0304-3959(00)00243-8] [PMID: 10779675]

[20] Chimenti PC, Hammert WC. Ulnar neuropathy at the elbow: an evidence-based algorithm. Hand Clin 2013; 29(3): 435-42. [http://dx.doi.org/10.1016/j.hcl.2013.04.013] [PMID: 23895724]

[21] Zlowodzki M, Chan S, Bhandari M, Kalliainen L, Schubert W. Anterior transposition compared with simple decompression for treatment of cubital tunnel syndrome. A meta-analysis of randomized, controlled trials. J Bone Joint Surg Am 2007; 89(12): 2591-8. [http://dx.doi.org/10.2106/JBJS.G.00183] [PMID: 18056489]

[22] Caliandro P, La Torre G, Padua R, Giannini F, Padua L. Treatment for ulnar neuropathy at the elbow. Cochrane Database Syst Rev 2012; 7: CD006839.

[PMID: 22786500]

[23] Watts AC, Bain GI. Patient-rated outcome of ulnar nerve decompression: a comparison of endoscopic and open in situ decompression. J Hand Surg Am 2012; 34: 1492-8.

[24] Zuo D, Zhou Z, Wang H, et al. Endoscopic versus open carpal tunnel release for idiopathic carpal tunnel syndrome: a meta-analysis of randomized controlled trials. J Orthop Surg 2015; 10: 12.

[http://dx.doi.org/10.1186/s13018-014-0148-6] [PMID: 25627324] 
[25] Vasiliadis H, Georgoulas P, Shrier I, et al. Endoscopic release for carpal tunnel syndrome. Cochrane Database Syst Rev $2014 ; 1$. [http://dx.doi.org/10.1002/14651858.CD008265.pub2]

(C) Chimenti et al.; Licensee Bentham Open.

This is an open access article licensed under the terms of the Creative Commons Attribution-Non-Commercial 4.0 International Public License (CC BY-NC 4.0) (https://creativecommons.org/licenses/by-nc/4.0/legalcode), which permits unrestricted, non-commercial use, distribution and reproduction in any medium, provided the work is properly cited. 